\title{
Validation of the Simplified Motor Score in patients with traumatic brain injury at a major trauma centre in South Africa
}

\author{
J J P Buitendag, ${ }^{1} \mathrm{MB} \mathrm{ChB}$; A Ras, ${ }^{1} \mathrm{MB} \mathrm{ChB}$; V Y Kong, ${ }^{1} \mathrm{MB} \mathrm{ChB}, \mathrm{MSc}, \mathrm{PhD}, \mathrm{MRCS}$; L L Bruce, ${ }^{1} \mathrm{MB} \mathrm{ChB}, \mathrm{FCS}(\mathrm{SA})$; \\ G L Laing, ${ }^{1} \mathrm{MB}$ ChB, MSc, PhD, FCS (SA); D L Clarke, ${ }^{1,2}$ MB ChB, MPhil, MBA, MSc, PhD; P Brysiewicz, ${ }^{3}$ BSocSc, BA, MCur, PhD \\ ${ }^{1}$ Pietermaritzburg Metropolitan Trauma Service, Department of Surgery, Nelson R Mandela School of Medicine, College of Health Sciences, \\ University of KwaZulu-Natal, Durban, South Africa \\ ${ }^{2}$ Department of Surgery, Faculty of Health Sciences, University of the Witwatersrand, Johannesburg, South Africa \\ ${ }^{3}$ School of Nursing and Public Health, College of Health Sciences, University of KwaZulu-Natal, Durban, South Africa
}

Corresponding author: J J P Buitendag (johan_buitendag@yahoo.com)

\begin{abstract}
Background. This study used data from a large prospectively entered database to assess the efficacy of the motor score (M score) component of the Glasgow Coma Scale (GCS) and the Simplified Motor Score (SMS) in predicting overall outcome in patients with traumatic brain injury (TBI). Objective. To safely and reliably simplify the scoring system used to assess level of consciousness of trauma patients in the acute setting. Methods. A retrospective observational review of the Pietermaritzburg Metropolitan Trauma Service hybrid electronic medical registry database was performed during the period January 2013 - December 2015. Patients were classified into three groups using their GCS as an injury severity score. These were mild TBI (GCS 13 - 15), moderate TBI (GCS 9 - 12) and severe TBI (GCS <9). The Glasgow M score was specifically evaluated to determine the relationship between the individual motor component and patient outcome.

Results. GCS scores and M scores were analysed in a total of 830 patients. There was a decline in survival rate when the M score on admission was $\leq 4$. The decline was more significant when the M score was $\leq 3$. Survival rates were $26.8 \%(11 / 41)$ for patients with an M score of 1,63.6\% (14/22) for those with a score of 2, 56.5\% (13/23) for those with a score of 3, 80.0\% (20/25) for those with a score of 4, and $95.5 \%$ (121/128) for those with a score of 5. Of 591 patients with an M score of 6, 580 (98.1\%) survived. Mortality rose dramatically with declining SMS. This was highly significant. When the M score was plotted against mortality in 830 patients, there was a correct prediction in 769 cases (accuracy 92.7\%, sensitivity 67.6\%, specificity 95\%). The area under the receiver operating characteristic (ROC) curve was 0.9037 , with a standard deviation (area) of 0.0227 . When comparing the SMS against mortality, the accuracy was $77.1 \%$, the sensitivity $84.5 \%$ and the specificity $76.4 \%$. The fitted ROC area was 0.891 and the empirical ROC area 0.86 .

Conclusion. The M score component of the GCS and the SMS accurately predict outcome in patients with TBI. In cases where the full GCS is difficult to assess, the M score and SMS can be used safely as a triage tool.
\end{abstract}

S Afr Med J 2018;108(2):90-93. DOI:10.7196/SAMJ.2018.v108i2.12757

The Lancet Commission on Global Surgery has shown that there is a huge unmet need for surgical and trauma care globally. ${ }^{[1]}$ According to the Commission, surgical conditions account for $\sim 30 \%$ of the global burden of disease, yet it is estimated that two-thirds of the world's population cannot access safe surgery and anaesthesia. This problem is especially acute in low- and middle-income countries. One of the major traumatic conditions on which such discrepancies impact is traumatic brain injury (TBI) ${ }^{[2-5]}$ The incidence of TBI in sub-Saharan Africa is 150 - 170 per 100000 compared with a global average of 106 per 100000 , yet the literature on the topic is relatively sparse, especially in comparison with that on other diseases with a high burden such as HIV and tuberculosis. ${ }^{[2-5]}$ It is not unreasonable to describe TBI as a forgotten and neglected epidemic, and trauma care in South Africa (SA) is perennially under-resourced for the burden of disease it is expected to manage. In addition, rural trauma care has major limitations in terms of human resources, infrastructure and equipment. ${ }^{[2-5]}$ Any system that increases complexity in such a setting is counterproductive, and any system that can reliably and safely simplify management must be supported. The Glasgow Coma Scale (GCS) is a clinical scoring system that after 40 years remains the cornerstone of the acute management and triage of patients with TBI. ${ }^{[6-8]}$ However, there is a growing concern that the traditional GCS suffers from needless complexity, which makes it less than ideal for managing large volumes of patients in resource-constrained settings. ${ }^{[9-12]} \mathrm{A}$ number of authors have proposed the use of an abbreviated score known as the Simplified Motor Score (SMS). ${ }^{[13,14]}$ Numerous studies have shown that the SMS reliably predicts the presence of TBI, the need for neurosurgical intervention and the need for intubation. Any system that reduces complexity in a strained environment without compromising quality of care deserves to be welcomed and encouraged.

\section{Objective}

In the light of the debate around the role and complexity and reliability of the GCS, we set out to review our experience with TBI and to compare the ability of the SMS, the individual motor score (M score) component of the GCS and the total GCS to predict the severity of TBI and the need for neurosurgery. We hoped to show that the SMS was as reliable and accurate as the GCS in predicting outcome in TBI. 


\section{Methods}

This was a retrospective review of a prospectively entered database undertaken at the Pietermaritzburg Metropolitan Trauma Service (PMTS), Pietermaritzburg, South Africa. The PMTS provides definitive trauma care to the city of Pietermaritzburg, the capital of KwaZulu-Natal (KZN) Province. It is one of the largest academic trauma centres in KZN and also serves as the referral centre for 19 other rural hospitals in the province, with a total catchment population of over three million. The hybrid electronic medical registry (HEMR) combines an electronic and paper-based patient record system with the facility of an electronic registry. All trauma patients in Pietermaritzburg are captured on this system.

The review was performed for the period January 2013 - December 2015. The GCS M score was specifically evaluated to determine the relationship between the individual motor component and patient outcome. The M score was compared with risk of death and need for surgery. We also determined the sensitivity and specificity of the total GCS in predicting the following outcomes: need for intubation, presence of a significant finding on computed tomography (CT), need for surgery, and mortality. We went on to derive the SMS for each patient based on the recorded motor component of the GCS. An SMS of 0 was considered to be equivalent to a GCS M score of 1 - 4, an SMS of 1 to an M score of 5, and an SMS of 2 to an M score of 6. We compared the SMS with the overall GCS score and with the GCS M score for the same outcomes as listed above.

\section{Statistical analysis}

We constructed receiver operating characteristic (ROC) curves and measured the areas under these curves to compare the predictive value of the GCS, M score and SMS against need for intubation, presence of a significant finding on CT, need for surgery, and mortality. We also calculated the $95 \%$ confidence intervals (CIs) of the areas under the curves for each outcome.

\section{Ethics approval}

Ethics approval for the study and for maintenance of the registry was obtained from the Biomedical Research Ethics Committee of the University of KwaZulu-Natal (ref. no. BE 207/09).

\section{Results}

A total of 830 patients were studied. Fig. 1 shows that there was a decline in survival rate when the $\mathrm{M}$ score on admission was $\leq 4$. The decline was more significant when the $\mathrm{M}$ score was $\leq 3$. Of a total of

\begin{tabular}{lllll}
\multicolumn{5}{l}{ Table 1 . M score v. survival } \\
\hline M score & Count, $\boldsymbol{N}$ & Survival, $\boldsymbol{n}$ & Died, $\boldsymbol{n}$ & Survival, $\%$ \\
\hline 1 & 41 & 11 & 30 & 26.8 \\
2 & 22 & 14 & 8 & 63.6 \\
3 & 23 & 13 & 10 & 56.5 \\
4 & 25 & 20 & 5 & 80.0 \\
5 & 128 & 121 & 7 & 94.5 \\
6 & 591 & 580 & 11 & 98.1 \\
M score = motor score. & & &
\end{tabular}

41 patients with an M score of 1, only 11 (26.8\%) survived. Survival rates were $63.6 \%(14 / 22)$ for those with a score of $2,56.5 \%(13 / 23)$ for those with a score of 3, 80.0\% (20/25) for those with a score of 4 , and $95.5 \%(121 / 128)$ for those with a score of 5 . Of 591 patients with a M score of 6, 580 (98.1\%) survived. Table 1 summarises these data, and Table 2 the data when the M score was converted to the SMS. Mortality rose dramatically with declining SMS, as shown in Fig. 2.

When plotting the $\mathrm{M}$ score against mortality, the prediction was correct in 769/830 patients (accuracy 92.7\%, sensitivity 67.6\%, specificity 95\%). The area under the ROC was 0.9037 with a standard deviation (SD) (area) of 0.0227. The ROC for M score and mortality was assessed. When comparing SMS score against mortality, the accuracy was $77.1 \%$, the sensitivity $84.5 \%$ and the specificity $76.4 \%$. The fitted ROC area was 0.891 and the empirical ROC area 0.86 . Table 3 breaks down the SMS and M score into their respective components and compares them with each outcome. Table 4 compares the M score and the SMS with all four outcomes (mortality, need for intubation, need for neurosurgery, and positive CT findings). The ROC curve for $\mathrm{M}$ score v. intubation was assessed. The accuracy was $92.9 \%$, the sensitivity $61.3 \%$ and the specificity $98.2 \%$. The fitted ROC area was 0.973 , the empirical ROC area 0.957 and the standard deviation (area) 0.0058 . When we compared SMS v. intubation, the accuracy was $84.8 \%$, the sensitivity $97.5 \%$ and the specificity $82.7 \%$. The fitted ROC area was 0.974 and the empirical ROC area 0.953 . The SD (area) was 0.0061 . Comparing M score v. neurosurgery, the accuracy was $71.2 \%$, the sensitivity $5.8 \%$ and the specificity $88.4 \%$. The fitted ROC area was 0.626 and the empirical ROC area 0.514. The SD (area) was 0.0320 . The ROC curve for SMS

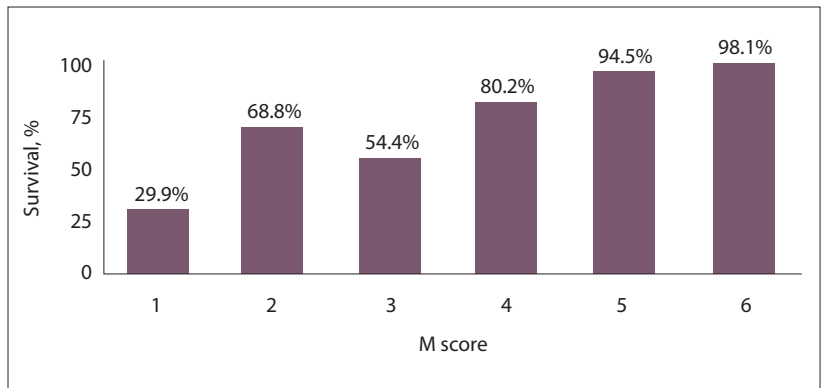

Fig. 1. M score v. survival. (M score = motor score. $)$

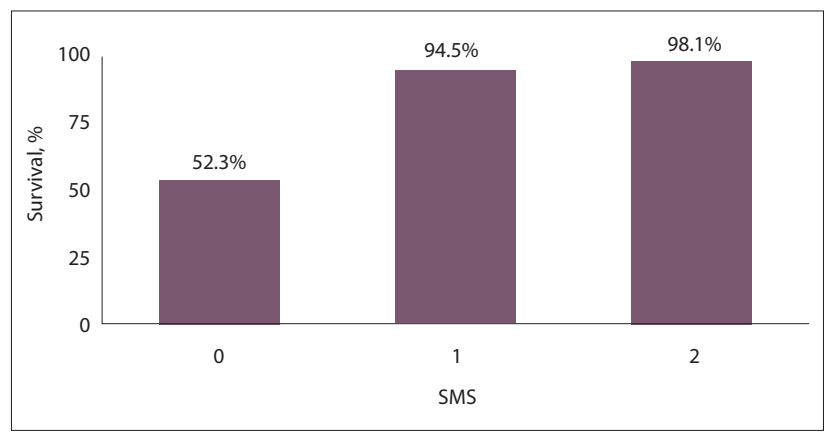

Fig. 2. SMS v. survival. (SMS = Simplified Motor Score. $)$

Table 2. SMS v. survival

\begin{tabular}{|c|c|c|c|c|c|c|}
\hline & SMS score & M score & Count, $N$ & Survival, $n$ & Died, $n$ & Survival, \% \\
\hline Obeys commands & 2 & 6 & 591 & 580 & 11 & 98.1 \\
\hline Localises pain & 1 & 5 & 128 & 121 & 7 & 94.5 \\
\hline Withdrawal to pain or less response & 0 & 4 & 111 & 58 & 53 & 52.3 \\
\hline
\end{tabular}


Table 3. SMS v. variables

\begin{tabular}{llllll}
\hline M score & Count, $\boldsymbol{N}$ & Intubation, $\boldsymbol{n}$ & Neuro. transfer, $\boldsymbol{n}$ & CT findings, $\boldsymbol{n}$ & SMS \\
\hline 1 & 41 & 37 & 1 & 41 & Withdrawal to pain or less \\
2 & 22 & 20 & 5 & 22 & response (SMS 0/GCS M $\leq 4)$ \\
3 & 23 & 16 & 4 & 21 & Localises pain (SMS 1/GCS M 5) \\
4 & 25 & 18 & 8 & 21 & Obeys commands (SMS 2/GCS M 6) \\
5 & 128 & 25 & 39 & 469 &
\end{tabular}

Table 4. Comparison of ROC curve findings with M score v. SMS

\begin{tabular}{|c|c|c|c|c|c|c|c|c|}
\hline & $\begin{array}{l}\text { M score v. } \\
\text { mortality }\end{array}$ & $\begin{array}{l}\text { SMS v. } \\
\text { mortality }\end{array}$ & $\begin{array}{l}\text { M score } v \text {. } \\
\text { intubation }\end{array}$ & $\begin{array}{l}\text { SMS v. } \\
\text { intubation }\end{array}$ & $\begin{array}{l}\text { M score v. } \\
\text { neurosurgery }\end{array}$ & $\begin{array}{l}\text { SMS v. } \\
\text { neurosurgery }\end{array}$ & $\begin{array}{l}\text { M score v. } \\
\text { CT findings }\end{array}$ & $\begin{array}{l}\text { SMS } \\
\text { v. CT } \\
\text { findings }\end{array}$ \\
\hline Cases, $N$ & 830 & 830 & 830 & 830 & 830 & 830 & 830 & 830 \\
\hline Correct, $n$ & 769 & 640 & 771 & 704 & 591 & 532 & 230 & 335 \\
\hline Accuracy, \% & 92.7 & 77.1 & 92.9 & 84.8 & 71.2 & 64.1 & 27.7 & 40.4 \\
\hline Sensitivity, \% & 67.6 & 84.5 & 61.3 & 97.5 & 5.8 & 32.9 & 12.3 & 31.2 \\
\hline Specificity, \% & 95 & 76.4 & 98.2 & 82.7 & 88.4 & 72.3 & 98.6 & 82.4 \\
\hline $\begin{array}{l}\text { Positive cases } \\
\text { missed, } n\end{array}$ & 23 & 11 & 46 & 3 & 163 & 116 & 598 & 469 \\
\hline $\begin{array}{l}\text { Negative cases } \\
\text { missed, } n\end{array}$ & 38 & 179 & 13 & 123 & 76 & 182 & 2 & 26 \\
\hline $\begin{array}{l}\text { Area under ROC } \\
\text { curve }\end{array}$ & 0.9037 & 0.8905 & 0.9734 & 0.9743 & 0.6258 & 0.6124 & 0.5043 & 0.5398 \\
\hline SD (area) & 0.0227 & 0.0334 & 0.0058 & 0.0061 & 0.0320 & 0.0368 & 0.0494 & 0.0590 \\
\hline Fitted ROC area & 0.904 & 0.891 & 0.973 & 0.974 & 0.626 & 0.612 & 0.504 & 0.54 \\
\hline Empirical ROC area & 0.873 & 0.86 & 0.957 & 0.953 & 0.514 & 0.517 & 0.578 & 0.576 \\
\hline
\end{tabular}

v. neurosurgery showed accuracy of $64.1 \%$, sensitivity of $32.9 \%$ and specificity of $72.3 \%$. The fitted ROC area was 0.612 and the empirical ROC area 0.517. The SD (area) was 0.0368 . We contend that this indicates that in a setting where resources are limited, most patients who are accepted by neurosurgery on the basis of the SMS will do well and have a good prognosis. The ROC curve of M score v. positive CT findings showed accuracy of $27.7 \%$, sensitivity of $12.3 \%$ and specificity of $98.6 \%$. The fitted ROC area was 0.504 and the empirical ROC area 0.578. The SD (area) was 0.0494. The ROC curves for SMS v. positive CT findings showed accuracy of $40.4 \%$, sensitivity of $31.2 \%$ and specificity of $82.4 \%$. The fitted ROC area was 0.54 and the empirical ROC area 0.576, with an SD (area) of 0.0590.

\section{Discussion}

Teasdale and Jennett first proposed the GCS in 1974 and modified it in subsequent publications. ${ }^{[6-8]}$ Since then the GCS has become ubiquitous, and it remains a clinical scoring system in an era when advanced imaging is increasingly used to assess patients and to predict outcome. However, more recently the GCS has been criticised for being confusing, unreliable and excessively complex. ${ }^{[9-11]}$ It has become apparent that the total score does not always accurately reflect a patient's condition. This is especially the case in trauma, where it may be difficult to assess verbal or eye response in a patient who is intubated or has suffered maxillofacial trauma. In the light of this, a number of authors have investigated whether individual components of the GCS are able to predict the outcome of TBI. ${ }^{[1-14]}$ The component most commonly studied has been the M score. The SMS has been proposed as a system that provides a meaningful, objective prognostic assessment of a patient's level of consciousness and is capable of predicting outcome. ${ }^{[11-14]}$ The SMS ignores the eye and verbal response and focuses exclusively on the $\mathrm{M}$ score, and is defined as follows: obeys commands $=2$, localises to pain $=1$, and withdraws to pain or less response $=0$. A patient with an SMS of $<2$ is at significantly increased risk of having a TBI that will require neurosurgical intervention or intubation, or that will cause death. As long ago as 1988, Choi et al. ${ }^{[9]}$ reported that using the patient's $\mathrm{M}$ score and age was as accurate and reliable as using the GCS in predicting outcome in these patients. ${ }^{[9]}$ Since then, several authors have reported that the M score is both sensitive and specific in predicting mortality from TBI. ${ }^{[11-14]}$ Our current data are very much in keeping with these findings, and this has major clinical implications. The development of the SMS provides a simplified but reliable tool to assess level of consciousness in trauma patients. Our current data and data from the international literature confirm that the SMS can predict key outcomes accurately. It is therefore a highly suitable and appropriate tool for use in our environment.

The resources available to manage the burden of trauma in SA are perennially insufficient. Triage is therefore a reality in our environment, and accurate prioritisation of trauma patients is essential. A triage tool that is accurate and easy to use is especially important in managing TBI, as patients often need to be transported over long distances for imaging and for management. ${ }^{[1,15,16]}$ The complexity of the traditional GCS and its variability make it particularly unsuited for use in a country like SA, where there is a significant degree of 
heterogeneity of experience and competence among staff in the field and in rural district hospitals. In addition, communication between staff at the receiving and referral institutions is often less than ideal, making a simplified score very desirable. The score that can be assessed in almost all patients, even those in the intensive care unit, is the $\mathrm{M}$ score. Our findings and those of authors in other countries confirm that the M score by itself and the SMS accurately predict mortality and outcome in patients with a TBI. Their use in trauma care in SA should be supported and encouraged.

Although on the basis of the ROCs both the M score and the SMS are poor predictors of CT findings and the need for neurosurgical intervention, it is unrealistic to expect the SMS to predict these clinical endpoints. The SMS is a triage tool for use in the field and in rural institutions to identify patients who need urgent resuscitation and referral to more appropriate levels of care. For this purpose, the SMS appears to be as accurate as the GCS.

\section{Study limitations}

This study, like most, has limitations. The data were only collected from a single centre. Some entries into the electronic database had missing data, and these patients could not be included. Misclassification bias should also be kept in mind and must be considered in all information that was entered by staff members.

\section{Conclusion}

This study confirms reports from international authors that the $\mathrm{M}$ score and the SMS can reliably predict a significant TBI as well as mortality. The simplicity of the scores and the ease with which they can be determined make them particularly suitable for use in our relatively austere prehospital and rural environments. Use of these scores in trauma care in SA should be supported and encouraged.

\section{Acknowledgements. None.}

Author contributions. JJPB, DLC and AR analysed the data and wrote the manuscript. VYK, JLB, GLL and PB reviewed the manuscript.
Funding. None.

Conflicts of interest. None.

1. Meara J, Greenberg S. The Lancet Commission on Global Surgery Global surgery 2030: Evidence and solutions for achieving health, welfare and economic development. Surgery 2015;157(5):834-835. https://doi.org/10.1016/j.surg.2015.02.009

2. Jerome E, Laing G, Bruce J, Sartorius B, Brysiewicz P, Clarke D. An audit of traumatic brain injury (TBI) in a busy developing-world trauma service exposes a significant deficit in resources available to manage severe TBI. S Afr Med J 2017;107(7):621-625. https://doi.org/10.7196/SAMJ.2017. v107i7.10562

3. Kong V, Bruce J, Sartorius B, et al. Civilian cerebral gunshot wounds in rural South African patients are associated with significantly higher mortality rates than in urban patients. Eur J Trauma Emerg Surg 2017. https://doi.org/10.1007/s00068-017-0800-z

4. Wong J, Linn K, Shinohara R, Mateen F. Traumatic brain injury in Africa in 2050: A modeling study. Eur J Neurol 2015;23(2):382-386. https://doi.org/10.1111/ene.12877

5. Hyder AA, Wunderlich CA, Puvanachandra P, Gururaj G, Kobusingye OC. The impact of traumatic Hyder AA, Wunderlich CA, Puvanachandra P, Gururaj G, Kobusingye OC.
brain injuries: A global perspective. NeuroRehabilitation 2007;22(5):341-353.

6. Jennett B, Teasdale G. Aspects of coma after severe head injury. Lancet 1977;309(8017):878-881. https://doi.org/10.1016/S0140-6736(77)91201-6

7. Teasdale G, Jennett B. Assessment of coma and impaired consciousness. Lancet 1974;304(7872):81-84. https://doi.org/10.1016/S0140-6736(74)91639-0

8. Teasdale G, Jennett B. Assessment and prognosis of coma after head injury. Acta Neurochir 1976;34(14):45-55. https://doi.org/10.1007/BF01405862

9. Choi S, Narayan R, Anderson R, Ward J. Enhanced specificity of prognosis in severe head injury. J Neurosurg 1988;69(3):381-385. https://doi.org/10.3171/ins.1988.69.3.0381

10. Green S. Cheerio, laddie! Bidding farewell to the Glasgow Coma Scale. Ann Emerg Med 2011;58(5):427430. https://doi.org/10.1016/j.annemergmed.2011.06.009

11. Gill M, Windemuth R, Steele R, Green S. A comparison of the Glasgow Coma Scale score to simplified alternative scores for the prediction of traumatic brain injury outcomes. Ann Emerg Med 2005;45(1):37-42. https://doi.org/10.1016/j.annemergmed.2004.07.429

12. Haukoos J, Gill M, Rabon R, Gravitz C, Green S. Validation of the Simplified Motor Score for the prediction of brain injury outcomes after trauma. Ann Emerg Med 2007;50(1):18-24. https://doi. org/10.1016/.annemergmed.2006.10.004

13. Gill M, Steele R, Windemuth R, Green S. A comparison of five simplified scales to the out-of-hospital Glasgow Coma Scale for the prediction of traumatic brain injury outcomes. Acad Emerg Med 2006;13(9):968-973. https://doi.org/10.1111/j.1553-2712.2006.tb00344.x

14. Thompson D, Hurtado T, Liao M, Byyny R, Gravitz C, Haukoos J. Validation of the Simplified Motor Score in the out-of-hospital setting for the prediction of outcomes after traumatic brain injury. Ann Emerg Med 2011;58(5):417-425. https://doi.org/10.1016/j.annemergmed.2011.05.033

15. Laing G, Bruce J, Skinner D, Allorto N, Clarke D, Aldous C. Development, implementation, and evaluation of a hybrid electronic medical record system specifically designed for a developing world surgical service. World J Surg 2013;38(6):1388-1397. https://doi.org/10.1007/s00268-013-2438-2

16. Laing G, Skinner D, Bruce J, Aldous C, Oosthuizen G, Clarke D. Understanding the burden and outcome of trauma care drives a new trauma systems model. World J Surg 2014;38(7):1699-1706. https://doi.org/10.1007/s00268-014-2448-8

Accepted 12 September 2017. 